\title{
Overexpression of RIP140 suppresses the malignant potential of hepatocellular carcinoma by inhibiting NF-кB-mediated alternative polarization of macrophages
}

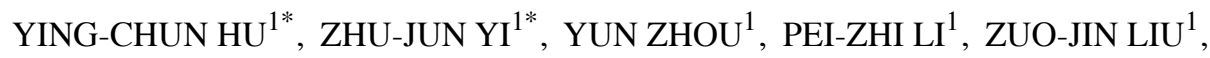 \\ SHI-GANG DUAN ${ }^{2}$ and JIAN-PING GONG ${ }^{1}$
}

\begin{abstract}
${ }^{1}$ Department of Hepatobiliary Surgery, The Second Affiliated Hospital of Chongqing Medical University, Chongqing 400010; ${ }^{2}$ Department of Hepatobiliary Surgery, The Ninth People's Hospital of Chongqing, Chongqing 400700, P.R. China
\end{abstract}

Received September 3, 2016; Accepted November 11, 2016

DOI: 10.3892/or.2017.5551

\begin{abstract}
Tumor-associated macrophages (TAMs) and their alternative activation contribute greatly to the development of hepatocellular carcinoma (HCC). Receptor-interacting protein 140 (RIP140) is widely expressed in macrophages and regulates macrophage-mediated energy metabolism, the inflammatory response and tumorigenesis. However, whether RIP140 is involved in the activation of TAMs has not been reported. In the present study, we determined the expression of RIP140 in macrophages after treatment with HCC-conditioned medium (HCM) for $24 \mathrm{~h}$. We also analyzed the effects of RIP140 overexpression on macrophage polarization, invasion and apoptosis of HepG2 and Huh7 cells. Transwell and apoptosis assays were used to estimated cell invasion and apoptosis. In addition, we investigated the effects of RIP140 overexpression in macrophages on the growth of $\mathrm{H} 22$ cells by subcutaneous injection of $\mathrm{H} 22$ cells along with macrophages in BALB/c nude mice. Western blotting and qRT-PCR were used to detect protein and mRNA expression associated with the NF- $\kappa \mathrm{B} / \mathrm{IL}-6$ axis in TAMs. Immunohistochemical and immunofluorescence staining were used to evaluate the protein expression of RIP140 or F4/80 in human HCC samples. The protein expression of RIP140 in peripheral blood mononuclear cells was detected by western blotting. Kaplan-Meier survival curve estimation of overall survival for patients with HCC was
\end{abstract}

Correspondence to: Dr Jian-Ping Gong, Department of Hepatobiliary Surgery, The Second Affiliated Hospital of Chongqing Medical University, Chongqing 400010, P.R. China

E-mail: gongjianping11@126.com

Dr Shi-Gang Duan, Department of Hepatobiliary Surgery, The Ninth People's Hospital of Chongqing, Chongqing 400700, P.R. China

E-mail: duanshigang@sina.com

*Contributed equally

Key words: hepatocellular carcinoma, RIP140, tumor-associated macrophages, invasion, polarization based on RIP140 or F4/80 expression in HCC samples. We found that HCM inhibited RIP140 expression and fostered the alternative activation of macrophages. RIP140 overexpression in TAMs significantly inhibited the alternative activation of macrophages by inhibiting the NF- $\mathrm{B} / \mathrm{IL}-6$ axis in TAMs, and suppressed $\mathrm{HCC}$ cell growth both in vitro and in vivo. In addition, the protein expression of RIP140 in peripheral blood monocytes was significantly lower in patients with HCC than in healthy people, and this result was consistent with the expression of RIP140 in HCC samples. Furthermore, low RIP140 expression and high F4/80 expression were found to be closely correlated with shorter survival time of the patients with HCC.

\section{Introduction}

Hepatocellular carcinoma (HCC), one of the most prevalent human cancers, is the third leading cause of cancer-related deaths worldwide. Although there are diverse treatments for HCC, such as surgical resection, radiofrequency ablation, radiotherapy and chemoembolization, the prognosis of patients with HCC remains poor (1-3). Thus, it is necessary to develop a deeper understanding of the molecular mechanisms to identify new molecular markers that can be used for early diagnosis and therapy of HCC, and to improve the outcome of these patients. Recent studies have focused on the important role of the tumor microenvironment in the progression, invasion and metastasis of HCC (4-6). Tumor-associated macrophages (TAMs) are the most abundant cellular component of the tumor microenvironment, and they play essential roles in the treatment of cancers, such as pancreatic cancer and HCC $(7,8)$. Previous studies have shown that TAMs are primarily characterized as alternatively activated M2-like macrophages, with high expression levels of peroxisome proliferator-activated receptor (PPAR), CD206 and arginase (Arg)-1, and low expression levels of tumor necrosis factor- $\alpha$ (TNF- $\alpha$ ). In addition, M2-like TAMs play leading roles in immune suppression by interacting with stromal cells, which greatly contributes to HCC growth, invasion and metastasis (9-11). These studies suggest that targeting the regulation of TAM polarization presents a potential therapeutic strategy for HCC. 
Receptor-interacting protein 140 (RIP140) is a nuclear receptor co-regulator that affects biological and pathological processes in the body, including energy metabolism, inflammatory response and tumorigenesis (12-14). Previous studies have found that RIP140-mediated macrophage polarization plays an essential role in regulating the inflammatory response. Overexpression of RIP140 in macrophages promoted macrophages to an M1-like polarization and expanded the inflammatory response. Conversely, lowering the level of RIP140 in macrophages not only reduced M1-like macrophages, but also expanded alternative polarization and promoted endotoxin tolerance (ET), which relieves the inflammatory response $(13,15,16)$. However, the role of RIP140 in TAMs remains completely unknown.

In the present study, we demonstrated that RIP140 expression in TAMs plays a role in the growth of hepatoma cells, which is closely related to the prognosis of patients with liver cancer. Our data, presented in the present study, identified RIP140 as a potential target in HCC immunotherapy that may promote macrophage-mediated antitumor immunity.

\section{Materials and methods}

Cell lines and cell cultures. Human HCC cell lines Huh7 and HepG2, and the mouse hepatoma cell line H22 were purchased from the American Type Culture Collection (ATCC, Rockville, MD, USA) and maintained in Dulbecco's modified Eagle's medium (DMEM; HyClone, Logan, UT, USA) with 10\% fetal bovine serum (FBS) and $1 \%$ penicillin $\mathrm{G}$ and streptomycin in $37^{\circ} \mathrm{C}$ humidified air containing $5 \% \mathrm{CO}_{2}$.

Human subjects and isolation of mononuclear cells. Human HCC tissues were obtained from 60 patients. Peripheral blood specimens were obtained from 5 healthy volunteers and 5 patients. All the patients were pathologically diagnosed with $\mathrm{HCC}$ at The Second Affiliated Hospital of Chongqing Medical University. Peripheral blood specimens were used for the isolation of peripheral mononuclear cells. Mononuclear cell isolation was performed as previously described (17). None of the individuals were positive for HCV or HIV. No chemotherapy, radiotherapy or surgical treatment was performed on these patients prior to obtaining blood specimens. Informed consent was obtained from all patients before the study was initiated. The present study was approved by the Research Ethics Committee of The Second Affiliated Hospital of Chongqing Medical University.

Macrophage preparation and lentivirus-mediated overexpression of RIP140. Peritoneal macrophages (PMs) were harvested from $\mathrm{BALB} / \mathrm{c}$ mice $72 \mathrm{~h}$ after intraperitoneal injection with $2 \mathrm{ml}$ of sterile $6 \%$ starch solution. The lentivirus-mediated PM overexpression vector containing RIP140 was constructed as previously described (12). Lentivirus packaging and cell transduction were carried out as previously described (18).

HCC-conditioned medium. $\mathrm{H} 22$ cells were cultured in serumfree DMEM for $24 \mathrm{~h}$. The supernatants were collected and used as HCC-conditioned medium (HCM). PMs were cultured with different amounts of HCM or exposed to HCM for different time periods in 6-well plates. Briefly, different amounts of HCM were added into complete medium to keep the final volume at $2,000 \mu 1$. Therefore, the final percentages of the $0,100,200$, 300 and $400 \mu \mathrm{l}$ of HCM were $0,5,10,15$ and $20 \%$, respectively.

In vivo tumorigenicity. Sixteen 4 -week-old BALB/c nude mice were housed in a pathogen-free environment and used for the HCC xenografts. Xenografts were prepared by subcutaneous injection of $\mathrm{H} 22$ cells along with homologous PMs transfected with Lv-GFP-RIP140 or Lv-GFP-NC at a ratio of 4:1. Four mice were sacrificed every week, and the volume and the weight of the tumors were calculated. All experimental procedures were conducted in accordance with the Guide for the Care and Use of Laboratory Animals and approved by our institutional ethical guidelines for animal experiments.

Cell apoptosis assay. $\mathrm{H} 22$ cells were harvested and washed with phosphate-buffered saline (PBS) 3 times after co-culture with PMs for $24 \mathrm{~h}$. Then, an apoptosis assay was performed using an Annexin V-FITC/PI Cell Apoptosis kit (KeyGen, Nanjing, Jiangsu, China). Briefly, a suspension (100 $\mu \mathrm{l})$ of $5 \times 10^{5} \mathrm{H} 22$ cells was incubated with $5 \mu 1$ of Annexin V and $1 \mu \mathrm{l}$ of propidium iodide (PI) at room temperature for $15 \mathrm{~min}$. The apoptotic rate was determined by flow cytometry (BD Pharmingen, San Diego, CA, USA).

Cell invasion assay. Cell invasion assays were performed in numerous studies. Briefly, $1 \times 10^{5}$ Huh7 or $1 \times 10^{5} \mathrm{HepG} 2$ cells were added to the upper chamber of an insert coated with Matrigel (BD Biosciences, San Jose, CA, USA). Cell invasion was allowed to proceed at $37^{\circ} \mathrm{C}$ for $24 \mathrm{~h}$. Then, the upper chambers were washed with PBS 3 times, and the cells were fixed with methanol at room temperature for $30 \mathrm{~min}$. After the cells on the inner surface of the filter membrane were removed, invading cells were stained with $0.1 \%$ crystal violet at room temperature for $15 \mathrm{~min}$, and washed again with PBS 3 times. Images were captured and cells were counted using digital microscopy.

RNA extraction and $q R T-P C R$ analysis. Total RNA was isolated from PMs using TRIzol reagent (Invitrogen) according to the manufacturer's protocol. qRT-PCR was performed using SYBR $^{\circledR}$-Green (Takara, Dalian, China) and an ABI Prism 7900 Sequence Detection System (Applied Biosystems, Foster City, CA, USA) according to the manufacturer's protocol. The primers used were as follows: CD206 forward, 5'-GGGAC TCTGGATTGGACTCA-3' and reverse, 5'-CCAGGCTCTGA TGATGGACT-3'; Arg-1 forward, 5'-CCCCAGTACCAACAG GACTACC-3' and reverse, 5'-TGAACGTGGCGGAATTTT GT-3'; PPAR forward, 5'-TCCCATACACAACCGCAGT CGC-3' and reverse, 5'-GGGGTCATTTGGTGACTCTGG GGT-3'; TNF- $\alpha$ forward, 5'-GGATCTCAAAGACAACCA AC-3' and reverse, 5'-ACAGAGCAATGACTCCAAAG-3'; NF- $\mathrm{B}$ forward, 5'-AGTGTGGAGGCTGCCTTGCGAATG-3' and reverse, 5'-TGGGCTTTCAAGACTGGAACGGTC-3'; GAPDH forward, 5'-CACCCACTCCTCCACCTTTG-3' and reverse, 5'-CCACCACCCTGTTGCTGTAG-3'. Data were normalized to the expression of GAPDH.

Western blot analysis. PM cells or peripheral blood monocytes were collected and lysed with radio-immunoprecipitation assay 
buffer [50 mM Tris- $\mathrm{HCl}(\mathrm{pH} 7.4), 150 \mathrm{mM} \mathrm{NaCl}, 1 \%$ (v/v) NP-40, 0.1\% (w/v) SDS, 0.5\% (w/v) sodium deoxycholate] containing protease inhibitors and phosphatase inhibitors, and cell lysates were centrifuged at $12,000 \times \mathrm{g}\left(4^{\circ} \mathrm{C}\right.$ for $\left.10 \mathrm{~min}\right)$. Total cellular protein $(80 \mu \mathrm{g})$ was mixed with a quarter of loading buffer [62.5 mM Tris- $\mathrm{HCl}$ (pH 6.8), $10 \%$ glycerol, $2 \%$ SDS, $2 \% \beta$-mercaptoethanol and bromophenol blue], boiled for $5 \mathrm{~min}$, and subjected to 8 or $10 \%$ SDS-PAGE. Proteins were transferred to polyvinylidene difluoride membranes (Millipore, Bedford, MA, USA). After the membranes were blocked with Tris-buffered saline containing $0.05 \%$ Tween-20 (TBST) and 5\% fat-free milk, the membrane was incubated overnight at $4^{\circ} \mathrm{C}$ with primary antibodies in TBST with $5 \%$ bovine serum albumin (BSA). The next day, the membranes were further incubated with the corresponding horseradish peroxidase-conjugated secondary antibody at $37^{\circ} \mathrm{C}$ for $1 \mathrm{~h}$ and then washed 3 times with TBST. Band signals were analyzed and scanned using Quantity One Software (Bio-Rad, Hercules, CA, USA) after incubation with an enhanced chemiluminescence reagent (Millipore). Anti-RIP140 (ab42126), anti-p-c-jun (ab32385) and anti-TGF- $\beta$ (ab31013) antibodies were purchased from Abcam (Cambridge, MA, USA); anti-p-p65 (\#3037), anti-p65 (\#8242), anti-TRAF3 (\#4729) and anti- $\beta$-actin (\#3700) antibodies were all from Cell Signaling Technology (CST; Danvers, MA, USA).

Immunohistochemical staining. F4/80 antibody is a standard macrophage marker. Immunohistochemical staining was performed as previously described (19). Briefly, human liver cancer $(n=60)$ and subcutaneous tumor tissues $(n=4)$ were fixed in formalin and embedded in paraffin, and $4-\mu \mathrm{m}$-thick consecutive sections were cut and mounted on glass slides. Then, the slides were first dewaxed in xylol and rehydrated in a 100,95 and $85 \%$ graded alcohol series, with antigen retrieval in $0.01 \mathrm{M}$ sodium citrate solution $98^{\circ} \mathrm{C}$ for $15 \mathrm{~min}$. Endogenous peroxidase activity was blocked with $3 \% \mathrm{H}_{2} \mathrm{O}_{2}$-methanol and normal goat serum for $30 \mathrm{~min}$. The human liver cancer tissues were incubated with RIP140 antibody (1:80; Abcam) or F4/80 antibody (1:50; Santa Cruz Biotechnology, Santa Cruz, CA, USA) at $4^{\circ} \mathrm{C}$ overnight, and the subcutaneous tumor tissues were incubated with proliferating cell nuclear antigen (PCNA) antibody (1:100; Cell Signaling, Boston, MA, USA) at $4^{\circ} \mathrm{C}$ overnight. The next day, the slides were washed 3 times with PBS and incubated with the appropriate biotin-labeled secondary antibody for 10-15 min. Under high-power magnification (x400), micrographs of 5 independent microscopic fields of the stained cells were screened and captured using a Leica DMLA light microscope (Leica Microsystems, Wetzlar, Germany).

Tissue immunofluorescence. The expression levels of RIP140 and F4/80 in human liver cancer tissues $(n=60)$ were measured by immunofluorescence. The experimental procedure was performed as previously described (5). Cell nuclei were stained with 4',6-diamidino-2-phenylindole (DAPI).

Statistical analysis. Statistical analyses were performed using SPSS 17.0 software (SPSS, Inc., Chicago, IL, USA). Differences were assessed for statistical significance by GraphPad Prism. All of the data are expressed as the means \pm SD. Statistical
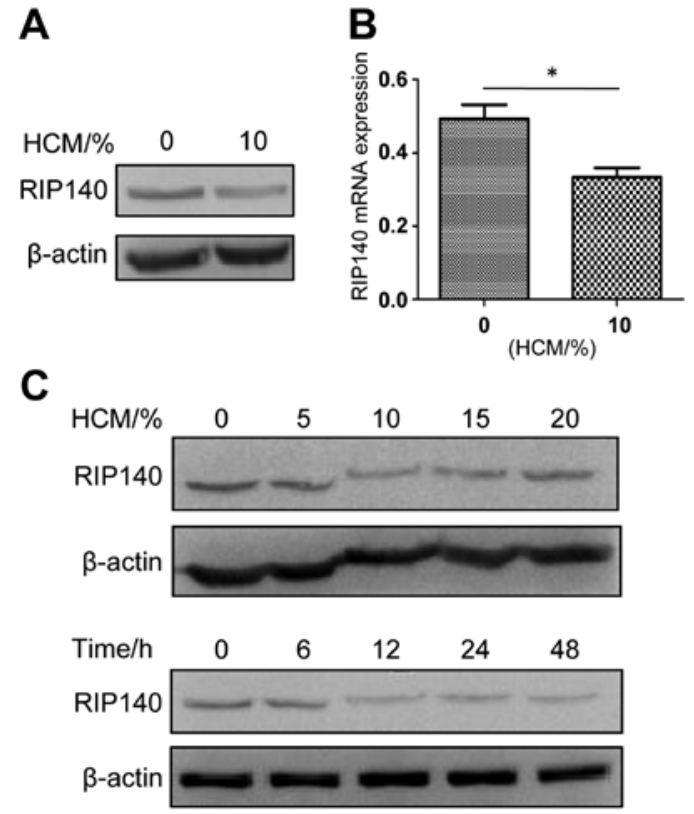

Figure 1. The HCC microenvironment inhibits RIP140 expression in TAMs (A-C) Western blotting was used to detect the protein level of RIP140 in PMs treated with HCM at different concentrations and for different lengths of time. (B) qRT-PCR was used to analyze the mRNA level of RIP140 in PMs with or without treatment with $10 \% \mathrm{HCM}$ for $24 \mathrm{~h}\left({ }^{*} \mathrm{P}<0.05\right)$. HCC, hepatocellular carcinoma; TAMs, tumor-associated macrophages; PMs, peritoneal macrophages; HCM, HCC-conditioned medium.

differences between two groups were determined by Student's t-test. The Kaplan-Meier method and log-rank test were used to perform the survival analysis. P-values of $<0.05$ were considered to indicate a statistically significant result.

\section{Results}

HCC microenvironment inhibits RIP140 expression in TAMs. $\mathrm{H} 22$ cells were cultured in serum-free DMEM for $24 \mathrm{~h}$. The supernatants were collected and used as HCM.

Preliminary experiments revealed that the protein (Fig. 1A) and mRNA (Fig. 1B) levels of RIP140 in PMs treated with $10 \% \mathrm{HCM}$ for $24 \mathrm{~h}$ were significantly lower than those of normal PMs. To evaluate the best length of HCM treatment and best concentration of HCM to induce the lowest expression of RIP140 in PMs, the protein level of RIP140 in PMs was examined by western blotting in concentration gradients (HCM 0, 5, 10, 15 and 20\%) and time gradients (time 0, 6, 12, 24 and 48 h) (Fig. 1C). As shown in Fig. 1C, the lowest level of RIP140 protein in TAMs was found in PMs treated with $10 \%$ $\mathrm{HCM}$ for $12 \mathrm{~h}$.

Overexpression of RIP140 in TAMs inhibits HCC microenvironment-mediated TAM M2-like polarization. Lentivirus transfection mediated RIP140 overexpression in PMs. To detect the efficiency of the lentivirus transfection, flow cytometry was used to analyze the rate of lentivirus transfection $96 \mathrm{~h}$ after PMs were transfected. A transfection rate of $\sim 60 \%$ was observed in the negative control (NC) lentiviral transfection, and a transfection rate of $63 \%$ was observed in the targeted lentiviral transfection (Fig. 2A). Western blotting was used to evaluate the protein expression level of RIP140 
A

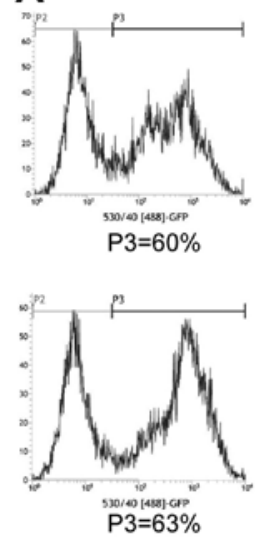

B

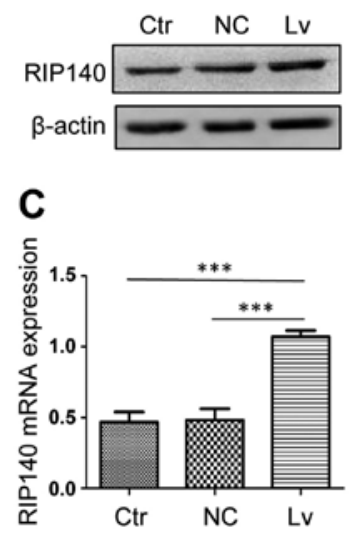

D

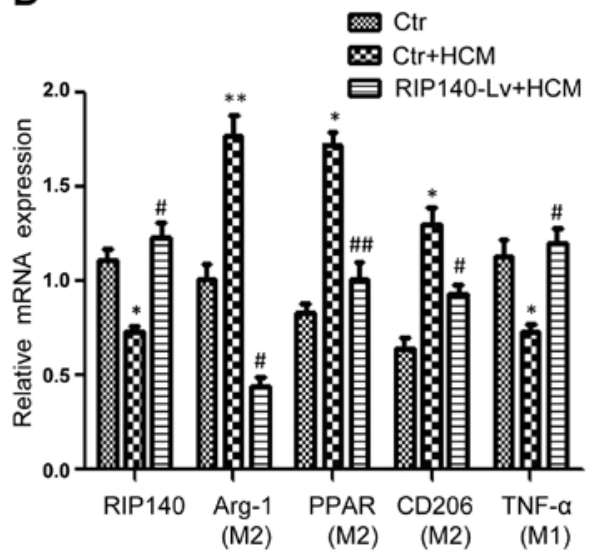

Figure 2. Lentivirus transfection induces RIP140 overexpression in PMs) (A) Flow cytometry was used to analyze the rate of lentivirus transfection. (B and C) The protein and mRNA levels of RIP140 in PMs with targeted lentivirus transfection were significantly higher than levels in the control groups. Overexpression of RIP140 in TAMs inhibited HCM-mediated TAM M2-like polarization and fostered TAM M1-like activation. (D) qRT-PCR was used to determine the mRNA expression of RIP140, Arg-1, PPAR, CD205 and TNF- $\alpha$. ${ }^{*} \mathrm{P}<0.05,{ }^{* *} \mathrm{P}<0.01$ and ${ }^{* * *} \mathrm{P}<0.001$, control + HCM group compared with control; ${ }^{~} \mathrm{P}<0.05$ and ${ }^{\# \#} \mathrm{P}<0.01$, RIP140 overexpressed + HCM group compared with control group. TAMs, tumor-associated macrophages; PMs, peritoneal macrophages; HCM, HCC-conditioned medium.

A
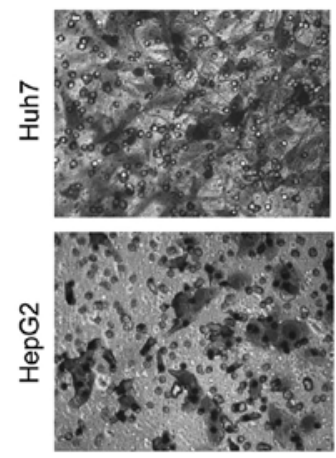

$\mathrm{Ctr}$

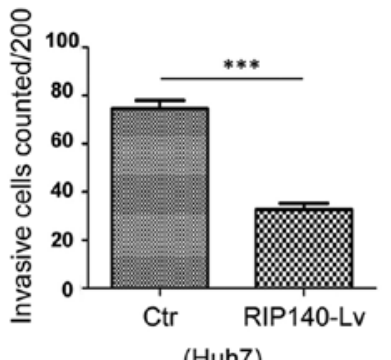

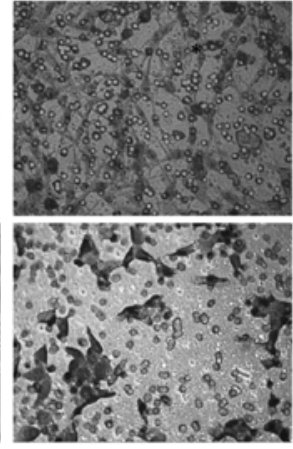

RIP140-Lv

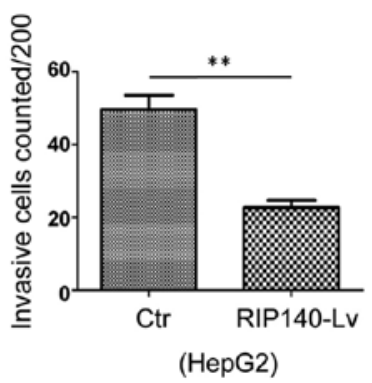

B

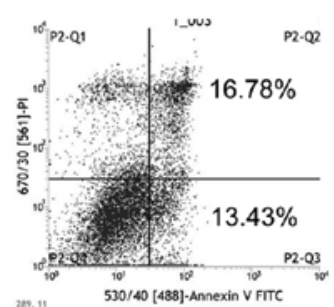

Ctr

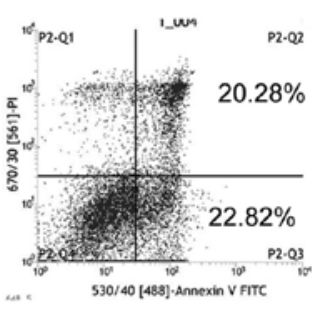

RIP140-Lv

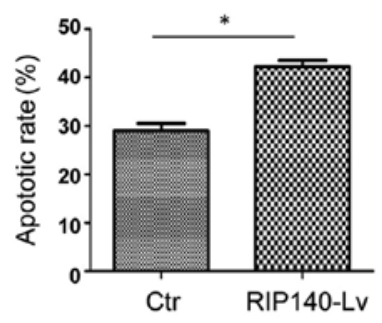

Figure 3. RIP140 overexpression suppresses the invasion and induces the apoptosis of hepatocarcinoma (HCC) cells. (A) Transwell assays were used to measure cell invasion. RIP140 overexpression inhibited the invasion of HepG2 and Huh7 cells. (B) Flow cytometry was used to determine the apoptosis rate of $\mathrm{H} 22$ cells. The percentage of early apoptotic cells is located in the lower right quadrant (Annexin V-FITC-positive/PI-negative) and the percentage of late apoptotic cells is located in the upper right quadrant (Annexin V-FITC-positive/PI-positive) $\left({ }^{*} \mathrm{P}<0.05,{ }^{* *} \mathrm{P}<0.01,{ }^{* * *} \mathrm{P}<0.001\right)$.

in PMs. As expected, RIP140 protein expression in PMs with targeted lentivirus transfection was significantly higher than that noted in the control groups (Fig. 2B). Using qRT-PCR, RIP140 mRNA expression was determined in the PMs. As expected, RIP140 mRNA expression in the PMs was also markedly increased in the targeted lentivirus transfection group (Fig. 2C).

Previous studies indicate that TAMs are primarily polarized to an M2-like phenotype in response to the tumor microenvironment (20). In addition, TAMs were reported to express high levels of Arg-1, PPAR and CD206 and low levels of the pro-inflammatory molecule TNF- $\alpha$ (8). To analyze the effects of RIP140 on TAMs, we overexpressed RIP140 in TAMs. qRT-PCR was used to detect the mRNA expression of RIP140, Arg-1, PPAR, CD205 and TNF- $\alpha$. As expected, overexpression of RIP140 in TAMs inhibited the expression of Arg-1, PPAR and CD206, which are closely related to the M2-like polarization phenotype of macrophages, and 
A

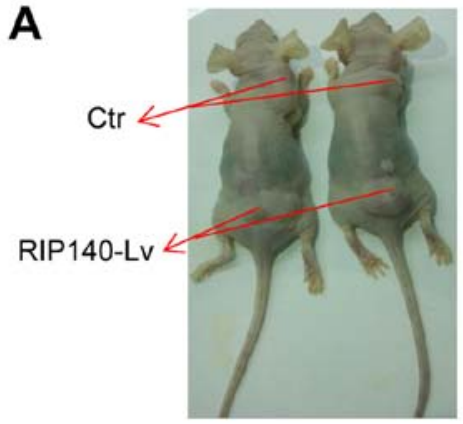

Ctr

B

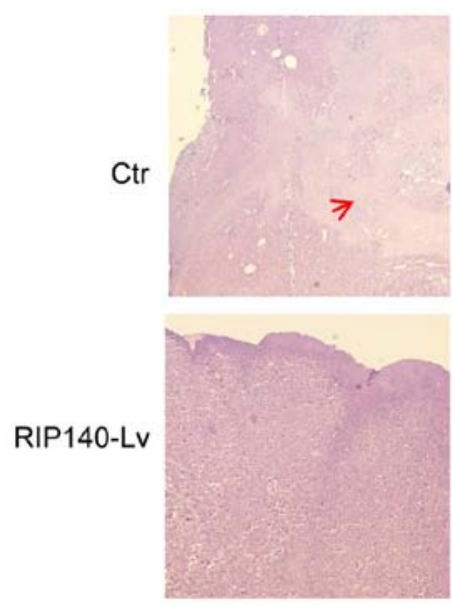

H\&E

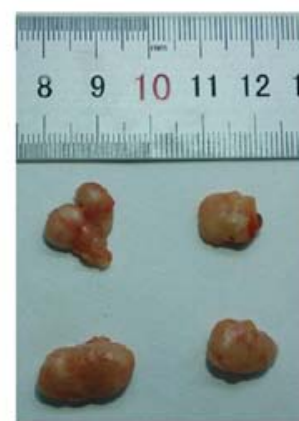

RIP140-Lv
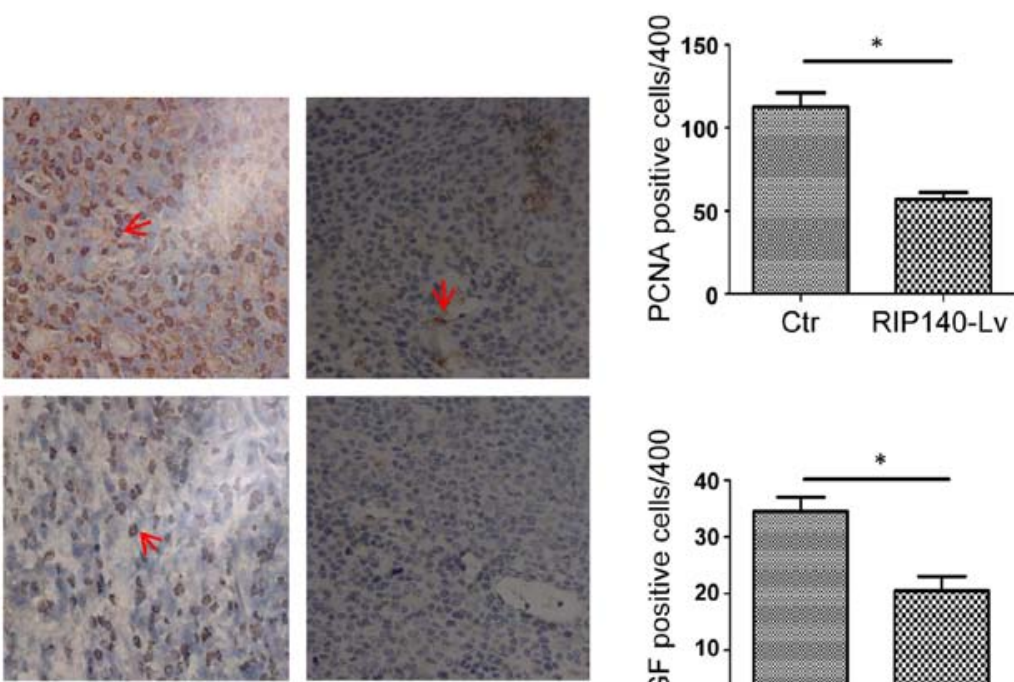

PCNA
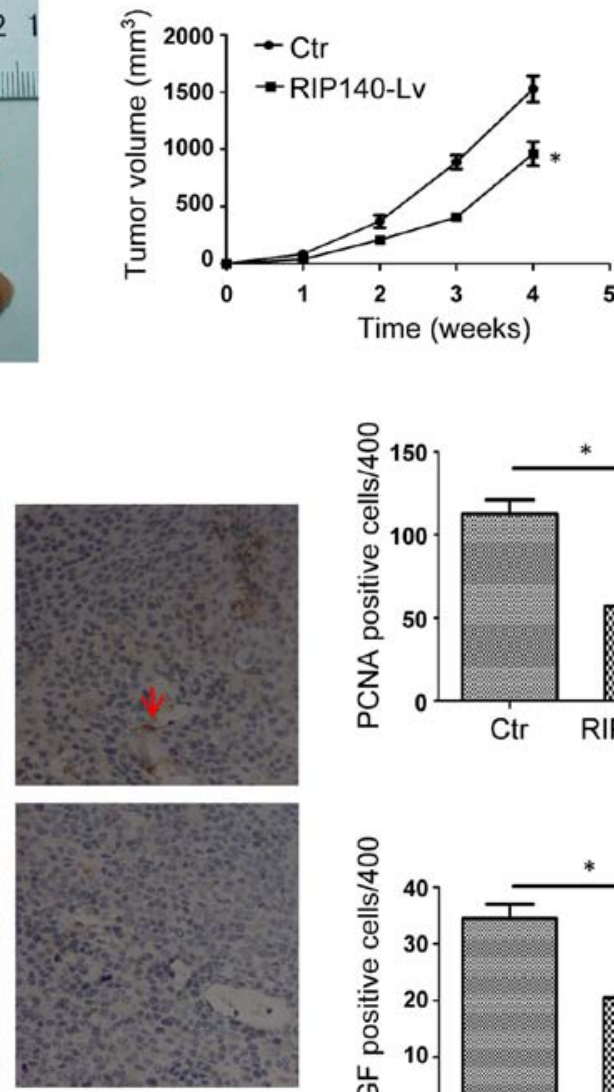

VEGF

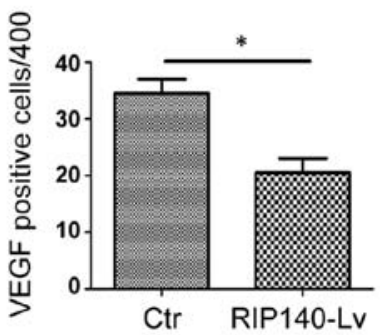

Figure 4. RIP140 overexpression in tumor-associated macrophages (TAMs) suppresses the growth of H22 subcutaneous tumors in BALB/c nude mice $(\mathrm{n}=16)$. (A) The weight and volume of the tumors at 4 weeks. (B) H\&E staining of tumor sections. Red arrows point to a necrotic area. Immunohistochemical staining detected PCNA and VEGF in tumor sections. Red arrows point to the PCNA- or VEGF-positive cells. Summary data are shown in the right panel $\left({ }^{*} \mathrm{P}<0.05\right)$.

promoted the expression of TNF- $\alpha$ (associated with the M1 phenotype) (Fig. 2D).

Overexpression of RIP140 in TAMs suppresses invasion and induces apoptosis of HCC cells. It has been well documented that M2-like TAMs promote HCC proliferation and metastasis $(8,9)$. In the present study, we found that overexpression of RIP140 in TAMs suppressed HCC microenvironmentmediated M2-like polarization of TAMs. To investigate whether RIP140 overexpressed in TAMs could inhibit migration and promote apoptosis of HCC cells, we performed Transwell assays and flow cytometric analysis. The Transwell assays further demonstrated that overexpression of RIP140 in TAMs greatly inhibited the invasion of HepG2 and Huh7 cells (Fig. 3A). Flow cytometry showed that overexpression of RIP140 in TAMs induced apoptosis in the H22 cells (Fig. 3B).

Overexpression of RIP140 in TAMs suppresses the growth of $\mathrm{H} 22$ subcutaneous tumors in $B A L B / c$ nude mice. To determine the effects of RIP140 in macrophages on tumor growth, we established a subcutaneous tumor model in mice $(n=16)$. Subcutaneous tumor models were prepared by subcutaneous injection of $\mathrm{H} 22$ cells combined with homologous PMs at a ratio of 4:1. As shown in Fig. 4A, compared with control macrophages, macrophages overexpressing RIP140 significantly inhibited the growth of H22 subcutaneous tumors. Consistently, the weight and volume of the tumors with RIP140-overexpressing macrophages were less than the weight and volume of tumors with control macrophages (Fig. 4A). Additionally, hematoxylin and eosin (H\&E) staining further demonstrated that overexpression of RIP140 in TAMs inhibited tumor growth compared with tumors in the control mice. We observed a large necrotic area (red arrows) in control tumor sections, which indicates that $\mathrm{H} 22$ cells rapidly proliferated (Fig. 4B). In addition, PCNA and vascular endothelial growth factor (VEGF) immunohistochemical staining in the RIP140-overexpressing tumor sections was significantly less than that in tumors of the control mice. The red arrows point to the PCNA- or VEGF-positive cells (Fig. 4B). Taken together, our data suggest that overexpression of RIP140 in TAMs inhibits HCC growth in vivo.

RIP140 overexpression in TAMs inhibits the growth of $H C C$ cell lines by suppressing the NF- $\kappa B / I L-6$ axis in TAMs. Numerous studies have reported that the tumor microenvironment activates the $\mathrm{NF}-\kappa \mathrm{B}$ pathway in TAMs, which is the well-known transcriptional controller of IL-6. The activation of the NF- $\mathrm{B} / \mathrm{IL}-6$ pathway in TAMs is closely 

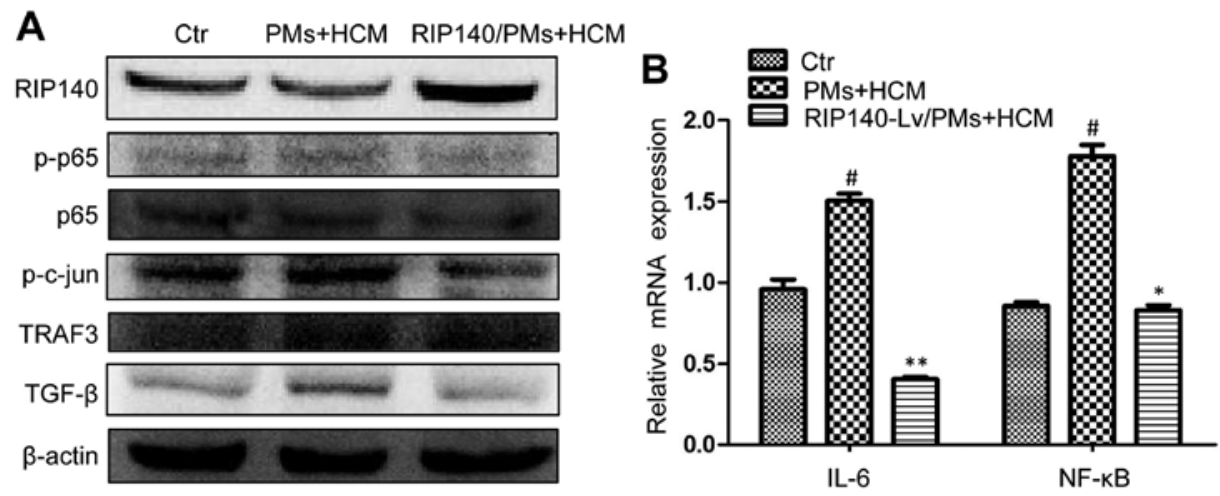

Figure 5. The tumor microenvironment activates the NF- $\kappa \mathrm{B} / \mathrm{IL}-6$ pathway and promotes TGF- $\beta$ expression in tumor-associated macrophages (TAMs). RIP140 overexpression inhibited TGF- $\beta$ and the NF- $\mathrm{kB} / \mathrm{IL}-6$ axis in TAMs. (A) Western blot analyses were performed to detect the indicated protein. (B) qRT-PCR was used to determine IL- 6 and the NF- $\mathrm{kB}$ mRNA expression in TAMs. ${ }^{*} \mathrm{P}<0.05$ and ${ }^{* *} \mathrm{P}<0.01$, RIP140 overexpression + HCM group compared with control group. ${ }^{"} \mathrm{P}<0.05$, control + HCM group compared with control group.
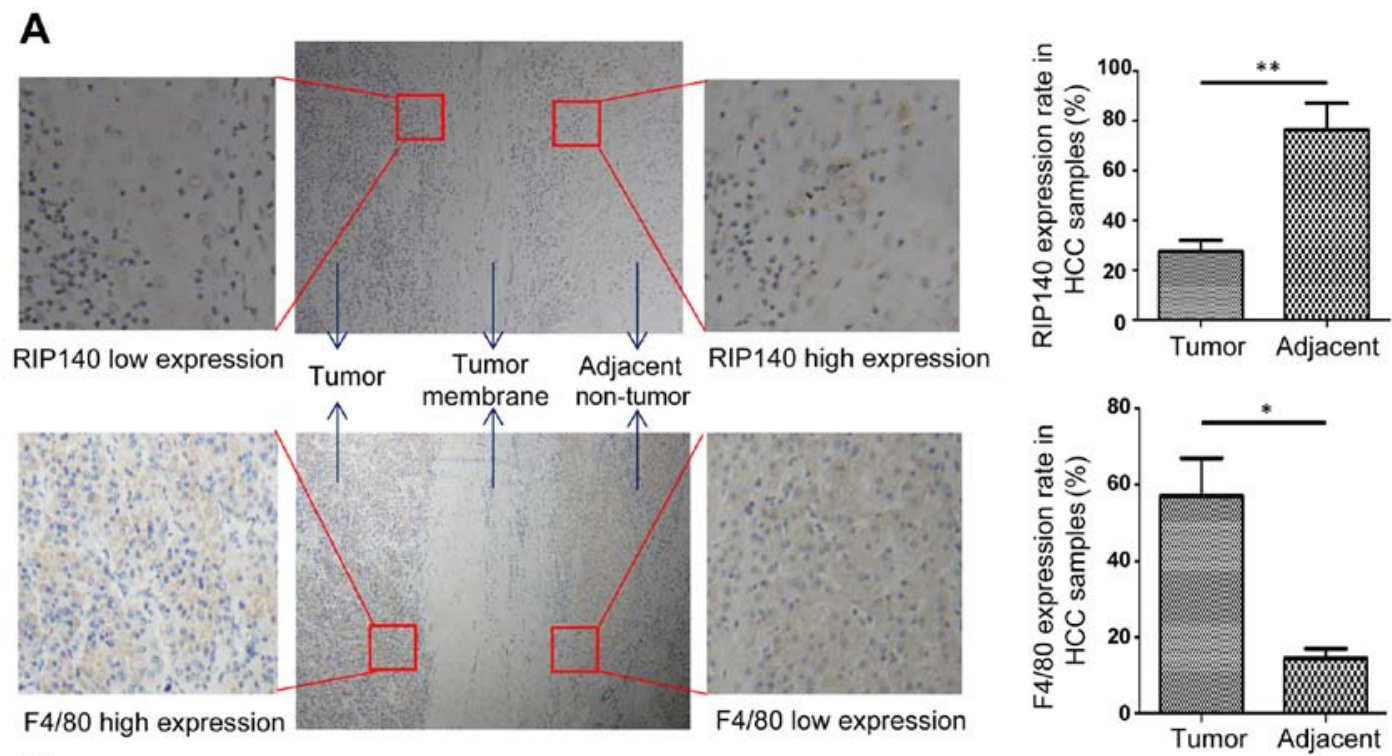

B

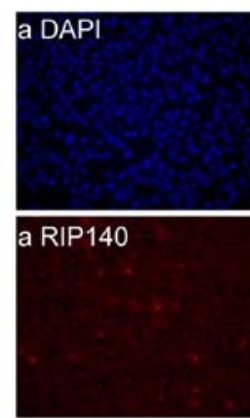

Adjacent
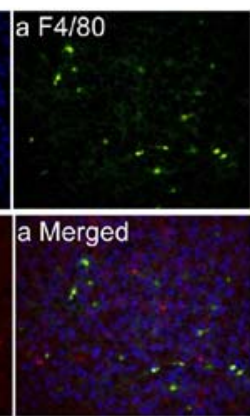

Non-tumor

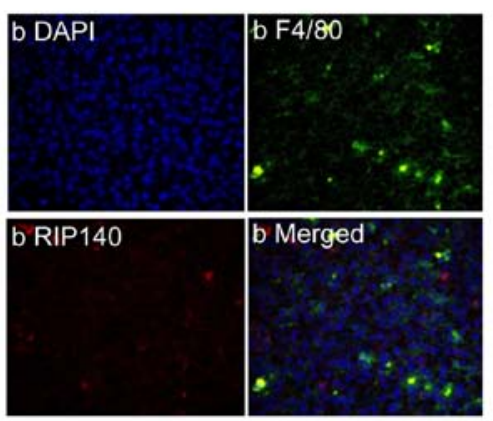

Tumor

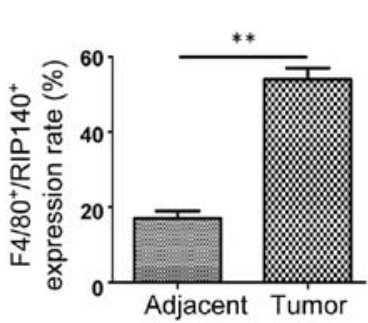

Figure 6. Low expression of RIP140 in tumor-associated macrophages (TAMs) of human hepatocellular (HCC) tissues. (A and B) Immunohistochemical and immunofluorescence were used to determine the expression of RIP140 and F4/80 protein in paraffin sections of human $\mathrm{HCC}$ samples $\left({ }^{*} \mathrm{P}<0.05\right.$, $\left.{ }^{* *} \mathrm{P}<0.01\right)$.

related to tumor growth (21-23). Therefore, we investigated the expression of the NF- $\mathrm{B} / \mathrm{IL}-6$ axis in RIP140-overexpressing TAMs. As expected, the tumor microenvironment activated the NF- $\kappa \mathrm{B} / \mathrm{IL}-6$ axis in TAMs and increased the protein expression of phosphorylated p65 (p-p65), phosphorylated c-Jun (p-c-Jun) and TRAF3 (Fig. 5A). Previous studies suggest that p-p65, p-c-Jun and TRAF3 are closely related to the NF- $\kappa \mathrm{B} / \mathrm{IL}-6$ axis and macrophage polarization $(24,25)$. Conversely, RIP140 overexpression in TAMs inhibited the NF- $\kappa \mathrm{B}-/ \mathrm{IL}-6$ axis and decreased the protein level of p-p65, p-c-Jun and TRAF3 (Fig. 5A). The IL-6 results were essentially in agreement with the above figures (Fig. 5B). This finding indicates that RIP140 overexpression in TAMs inhibited the growth of HCC cell lines probably by suppressing 
A
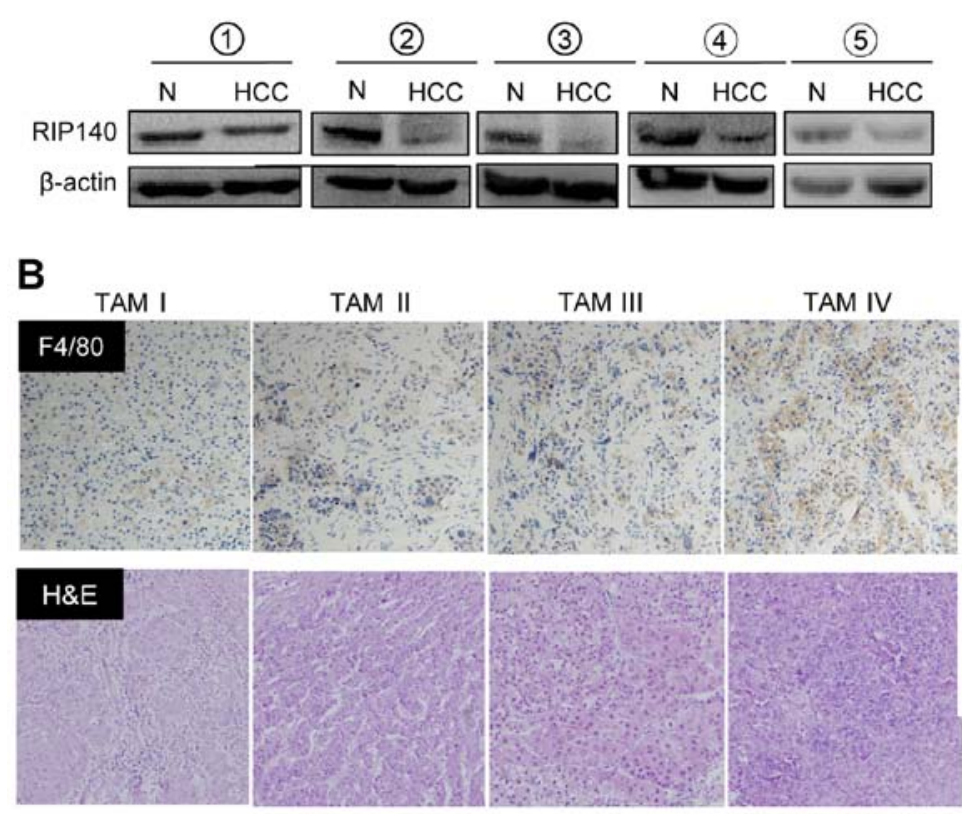
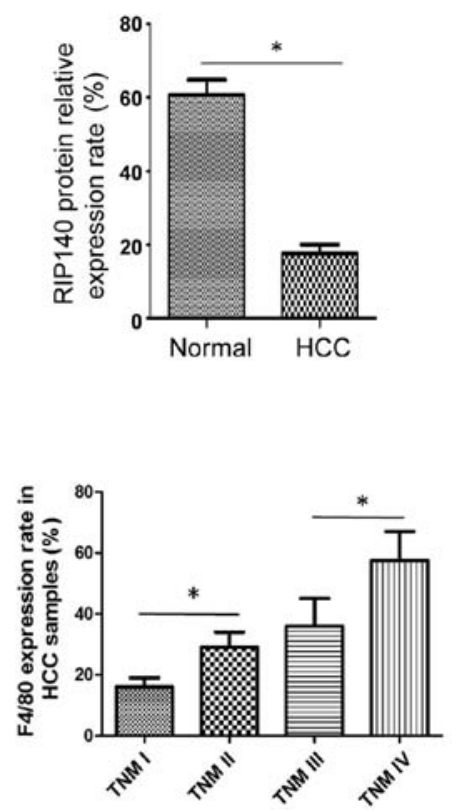

C
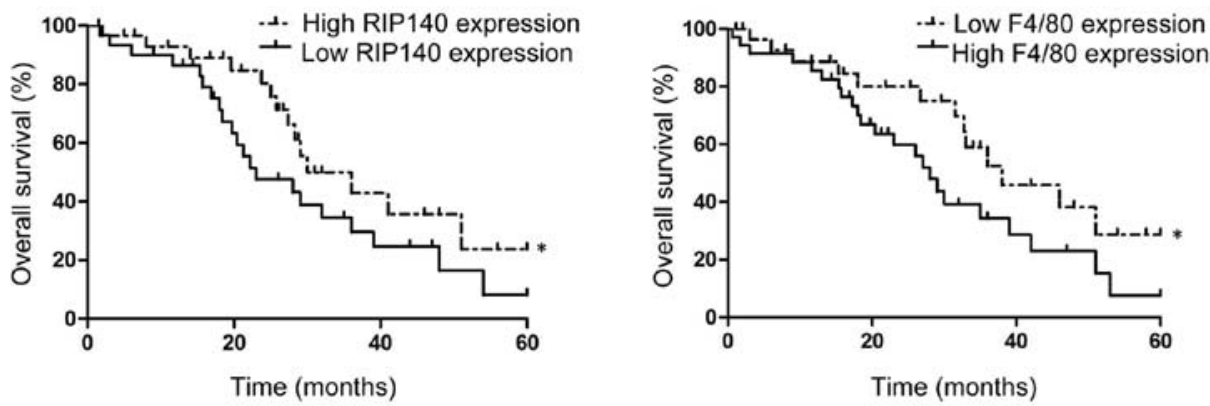

Figure 7. Low expression of RIP140 in peripheral mononuclear cells is a predictor of poor patient survival. (A) Western blot analyses were used to evaluate the protein level of RIP140 in human peripheral mononuclear cells. (B) F4/80 immunohistochemical staining and H\&E staining in different TNM stages. (C) Kaplan-Meier survival curve of overall survival based on RIP140 and F4/80 expression in patients with HCC ( $\mathrm{n}=60$ ). The patients with HCC were grouped according to the RIP140 and F4/80 expression results: high RIP140 expression (n=17) and low RIP140 expression (n=43) groups or high F4/80 expression $(n=38)$ and low F4/80 expression $(n=22)$ groups. The association between RIP140 or F4/80 expression and survival was analyzed using the Kaplan-Meier method $\left({ }^{*} \mathrm{P}<0.05\right)$.

the NF- $\mathrm{B}$ /IL-6 signaling pathway in TAMs. Transforming growth factor (TGF)- $\beta$ is closely related to tumor progression and metastasis (26). However, whether TGF- $\beta$ is a tumor promoter or suppressor is still unknown $(27,28)$. It has been reported that TGF- $\beta$ in the tumor microenvironment facilitates the alternative activation of macrophages and promotes tumor growth $(8,29)$. In the present study, we detected TGF- $\beta$ protein expression in TAMs. As shown, RIP140 overexpression decreased the tumor microenvironment-stimulated TGF- $\beta$ expression in TAMs (Fig. 5A). Therefore, we hypothesize that the TGF- $\beta$-promoted tumor growth was not only related to the TGF- $\beta$ secreted by tumor cells but was also related to the high expression of TGF- $\beta$ in TAMs. Of course, this hypothesis needs further confirmation.

Low expression of RIP140 in TAMs of human HCC tissues. To detect the expression of RIP140 and F4/80 in human HCC samples, the paraffin sections of human HCC tissues $(n=60)$ were assessed by immunohistochemistry and immunofluorescence (Fig. 6A and B). As shown in Fig. 6A, the protein expression of RIP140 in the cancer tissues was lower than that noted in adjacent non-tumor tissue. In contrast, the protein expression of F4/80 in cancer tissues was higher than that noted in adjacent non-tumor tissue. The expression level of RIP140 in macrophages was scored by evaluating the number of RIP140+-F4/80+ cells (both positive for RIP140 and F4/80) in relation to the total number of cells. As shown in Fig. 6B, the expression of RIP140 in macrophages (F4/80-positive cells) was significantly lower in the HCC specimens than that noted in adjacent non-tumor specimens, indicating low expression of RIP140 in TAMs of human HCC samples.

Low expression levels of RIP140 in peripheral mononuclear cells of patients with HCC predict poor patient survival. To detect the RIP140 protein expression in TAMs from HCC samples, we isolated the peripheral mononuclear cells from 5 patients with pathologically confirmed HCC at the Second Affiliated Hospital of Chongqing Medical University and 5 healthy volunteers. Western blot analyses were used to evaluate the RIP140 protein level in peripheral mononuclear 
cells. As expected, RIP140 protein expression in peripheral mononuclear cells from patients with HCC was significantly lower than that from healthy subjects (Fig. 7A). In fact, the positive expression rate of $\mathrm{F} 4 / 80$ in human HCC samples increased with the TNM stage (Fig. 7B). This indicates that a large number of macrophages with low RIP140 expression accumulate in HCC tissues. In addition, low RIP140 expression and high F4/80 expression were found to be closely correlated with shorter survival time (Fig. 7C).

\section{Discussion}

Tumor-associated macrophages (TAMs) and their alternative activation contribute greatly to the development of HCC (8-10). RIP140 is a nuclear receptor co-regulator that is widely expressed in macrophages and regulates macrophage-mediated energy metabolism, inflammatory response and tumorigenesis (12-16). However, whether RIP140 is involved in the activation and function of TAMs has not yet been reported. In the present study, for the first time, we found decreased expression of RIP140 in TAMs after treatment with an HCC microenvironment, which facilitated alternative activation of the macrophages and accelerated tumor growth. Our data provide a previously unrecognized link between RIP140 and TAM-related inflammation in HCC, and mark RIP140 as a potential target in $\mathrm{HCC}$ immunotherapy that may promote macrophage-mediated antitumor immunity. The present study presents the following evidence to support these conclusions.

Firstly, we found that HCC-conditioned medium (HCM) inhibited RIP140 expression and fostered the alternative activation of macrophages. Previous studies have found that RIP140 mediated macrophage polarization, which plays essential roles in regulating the inflammatory response $(13,14)$. Overexpression of RIP140 in macrophages promoted macrophages to M1-like polarization and expand the inflammatory response. Conversely, lowering the level of RIP140 in macrophages not only reduces M1-like macrophages but also expands alternative polarization, which promotes endotoxin tolerance (ET) and relieves inflammation $(13,15,16)$. We found that HCM decreased the expression of RIP140 in TAMs. Therefore, we suspected that the HCC microenvironment-mediated macrophage M2-like polarization may be closely related to the low expression of RIP140 in macrophages. To verify the above hypothesis, lentiviral-mediated transfection was performed to induce RIP140 overexpression in TAMs.

Secondly, RIP140 overexpression in TAMs significantly inhibited the alternative activation of macrophages and suppressed HCC cell growth both in vitro and in vivo. Visibly, the effect of the tumor microenvironment promoted TAMs to M2-like polarization and was closely related to decrease RIP140 expression in TAMs. Therefore, one important question is how RIP140 regulates TAM polarization and further affects tumor growth. Previous studies have shown that the TAM effect on the growth of tumors is associated with a TAM-mediated tumor microenvironment immune response, particularly related to the activation of the NF- $\mathrm{kB} / \mathrm{IL}-6$ axis. The high level of IL- 6 that induces 'parainflammation' in the tumor microenvironment is the most important reason for the promotion of tumor growth by TAMs (30-32). Our data revealed that overexpression of RIP140 in TAMs suppressed the NF- $\mathrm{KB} / \mathrm{IL}-6$ axis and reduced the release of IL-6 into the tumor microenvironment, which is one of the important reasons why RIP140 inhibited the growth of HCC in vivo and in vitro.

Finally, in order to evaluate RIP140 clinical values, we performed various related experiments using tissue and blood samples of patients with $\mathrm{HCC}$ or healthy volunteers. Our data indicated that RIP140 was poorly expressed in TAMs of human HCC tissues, which predicted poor patient survival. In the present study, we reproduced the findings regarding human peripheral mononuclear cells in TAMs (9). However, we think that our methods may not be precise. The best method is to directly extract the TAMs from fresh HCC tissues or adjacent non-tumor tissues to perform further studies (11). Due to technical reasons and limited experimental conditions, this research was not able to be satisfactorily completed.

In conclusion, our results demonstrated that the tumor microenvironment inhibits the transcription of RIP140 in TAMs, which promotes M2-like TAM polarization and HCC growth. Overexpression of RIP140 in TAMs suppresses HCC growth in vivo and in vitro. In addition, we found that there is a low expression level of RIP140 in TAMs of human HCC tissues, which predicts poor patient survival. These findings suggest that RIP140 plays a role in TAMs and may provide a new strategy for HCC treatment.

\section{Acknowledgements}

The present study was supported by the National Natural Science Foundation of China (nos. 31370753, 81470899 and 81401622), the Key Item of Chongqing Health and Family Planning Commission of China (2015zdxm026), and the Basic Science and Frontier Technology Research Foundation of Chongqing Science and Technology Commission (cstc2015jcy jBX0070).

\section{References}

1. Bruix J, Gores GJ and Mazzaferro V: Hepatocellular carcinoma: Clinical frontiers and perspectives. Gut 63: 844-855, 2014.

2. Schlachterman A, Craft WW Jr, Hilgenfeldt E, Mitra A and Cabrera R: Current and future treatments for hepatocellular carcinoma. World J Gastroenterol 21: 8478-8491, 2015.

3. Cheng AL, Thongprasert S, Lim HY, Sukeepaisarnjaroen W, Yang TS, Wu CC, Chao Y, Chan SL, Kudo M, Ikeda M, et al: Randomized, open-label phase 2 study comparing frontline dovitinib versus sorafenib in patients with advanced hepatocellular carcinoma. Hepatology 64: 774-784, 2016.

4. Zhou SL, Zhou ZJ, Hu ZQ, Huang XW, Wang Z, Chen EB, Fan J, Cao Y, Dai Z and Zhou J: Tumor-associated neutrophils recruit macrophages and T-regulatory cells to promote progression of hepatocellular carcinoma and resistance to sorafenib. Gastroenterology 150: 1646-1658.e17, 2016.

5. Ye LY, Chen W, Bai XL, Xu XY, Zhang Q, Xia XF, Sun X, Li GG, Hu QD, Fu QH, et al: Hypoxia-induced epithelial-tomesenchymal transition in hepatocellular carcinoma induces an immunosuppressive tumor microenvironment to promote metastasis. Cancer Res 76: 818-830, 2016.

6. Hernandez-Gea V, Toffanin S, Friedman SL and Llovet JM: Role of the microenvironment in the pathogenesis and treatment of hepatocellular carcinoma. Gastroenterology 144: 512-527, 2013.

7. Nywening TM, Wang-Gillam A, Sanford DE, Belt BA, Panni RZ, Cusworth BM, Toriola AT, Nieman RK, Worley LA, Yano M, et al: Targeting tumour-associated macrophages with CCR2 inhibition in combination with FOLFIRINOX in patients with borderline resectable and locally advanced pancreatic cancer: A single-centre, open-label, dose-finding, non-randomised, phase 1b trial. Lancet Oncol 17: 651-662, 2016. 
8. Flecken T and Sarobe P: Tim-3 expression in tumour-associated macrophages: A new player in HCC progression. Gut 64: 1502-1503, 2015.

9. Yan W, Liu X, Ma H, Zhang H, Song X, Gao L, Liang X and Ma C: Tim-3 fosters HCC development by enhancing TGF- $\beta$-mediated alternative activation of macrophages. Gut 64: 1593-1604, 2015.

10. Zhou W, Ke SQ, Huang Z, Flavahan W, Fang X, Paul J, Wu L, Sloan AE, McLendon RE, Li X, et al: Periostin secreted by glioblastoma stem cells recruits M2 tumour-associated macrophages and promotes malignant growth. Nat Cell Biol 17: 170-182, 2015.

11. Heusinkveld M and van der Burg SH: Identification and manipulation of tumor associated macrophages in human cancers. J Transl Med 9: 216, 2011.

12. Lapierre M, Bonnet S, Bascoul-Mollevi C, Ait-Arsa I, Jalaguier S, Del Rio M, Plateroti M, Roepman P, Ychou M, Pannequin J, et al: RIP140 increases APC expression and controls intestinal homeostasis and tumorigenesis. J Clin Invest 124: 1899-1913, 2014.

13. Ho PC, Tsui YC, Feng X, Greaves DR and Wei LN: NF-кBmediated degradation of the coactivator RIP140 regulates inflammatory responses and contributes to endotoxin tolerance. Nat Immunol 13: 379-386, 2012.

14. Nautiyal J, Christian M and Parker MG: Distinct functions for RIP140 in development, inflammation, and metabolism. Trends Endocrinol Metab 24: 451-459, 2013.

15. Liu PS, Lin YW, Burton FH and Wei LN: M1-M2 balancing act in white adipose tissue browning - a new role for RIP140. Adipocyte 4: 146-148, 2015.

16. Lin YW, Lee B, Liu PS and Wei LN: Receptor-interacting protein 140 orchestrates the dynamics of macrophage $\mathrm{m} 1 / \mathrm{m} 2$ polarization. J Innate Immun 8: 97-107, 2016

17. Moreli JB, Santos JH, Lorenzon-Ojea AR, Corrêa-Silva S, Fortunato RS, Rocha CR, Rudge MV, Damasceno DC, Bevilacqua $\mathrm{E}$ and Calderon IM: Hyperglycemia differentially affects maternal and fetal DNA integrity and DNA damage response. Int J Biol Sci 12: 466-477, 2016.

18. Feng X, Krogh KA, Wu CY, Lin YW, Tsai HC, Thayer SA and Wei LN: Receptor-interacting protein 140 attenuates endoplasmic reticulum stress in neurons and protects against cell death. Nat Commun 5: 4487-4495, 2014.

19. Lu X, Zhou C, Li R, Liang Z, Zhai W, Zhao L and Zhang S: Critical role for the long non-coding RNA AFAP1-AS1 in the proliferation and metastasis of hepatocellular carcinoma. Tumour Biol 37: 9699-9707, 2016
20. Mantovani A, Allavena P, Sica A and Balkwill F: Cancer-related inflammation. Nature 454: 436-444, 2008.

21. Mancino A and Lawrence T: Nuclear factor-kappaB and tumorassociated macrophages. Clin Cancer Res 16: 784-789, 2010.

22. Chang CP, Su YC, Lee PH and Lei HY: Targeting NFKB by autophagy to polarize hepatoma-associated macrophage differentiation. Autophagy 9: 619-621, 2013.

23. Wan S, Zhao E, Kryczek I, Vatan L, Sadovskaya A, Ludema G, Simeone DM, Zou W and Welling TH: Tumor-associated macrophages produce interleukin 6 and signal via STAT3 to promote expansion of human hepatocellular carcinoma stem cells. Gastroenterology 147: 1393-1404, 2014.

24. Hefetz-Sela S, Stein I, Klieger Y, Porat R, Sade-Feldman M, Zreik F, Nagler A, Pappo O, Quagliata L, Dazert E, et al: Acquisition of an immunosuppressive protumorigenic macrophage phenotype depending on c-Jun phosphorylation. Proc Natl Acad Sci USA 111: 17582-17587, 2014.

25. Lalani AI, Moore CR, Luo C, Kreider BZ, Liu Y, Morse HC III and Xie P: Myeloid cell TRAF3 regulates immune responses and inhibits inflammation and tumor development in mice. J Immunol 194: 334-348, 2015.

26. Zhu H, Luo H, Shen Z, Hu X, Sun L and Zhu X: Transforming growth factor- $\beta 1$ in carcinogenesis, progression, and therapy in cervical cancer. Tumour Biol 37: 7075-7083, 2016.

27. David CJ, Huang YH, Chen M, Su J, Zou Y, Bardeesy N, Iacobuzio-Donahue CA and Massagué J: TGF- $\beta$ tumor suppression through a lethal EMT. Cell 164: 1015-1030, 2016

28. Xu J, Acharya S, Sahin O, Zhang Q, Saito Y, Yao J, Wang H, Li P, Zhang L, Lowery FJ, et al: 14-3-3 $\zeta$ turns TGF- $\beta$ 's function from tumor suppressor to metastasis promoter in breast cancer by contextual changes of Smad partners from p53 to Gli2. Cancer Cell 27: 177-192, 2015.

29. Gratchev A: TGF- $\beta$ signalling in tumour associated macrophages. Immunobiology 222: 75-81, 2017.

30. Wu L, Zhang X, Zhang B, Shi H, Yuan X, Sun Y, Pan Z, Qian H and $\mathrm{Xu} \mathrm{W}$ : Exosomes derived from gastric cancer cells activate $\mathrm{NF}-\kappa \mathrm{B}$ pathway in macrophages to promote cancer progression. Tumour Biol 37: 12169-12180, 2016.

31. Karin M: NF-kappaB as a critical link between inflammation and cancer. Cold Spring Harb Perspect Biol 1: a000141, 2009.

32. Ara $T$ and Declerck YA: Interleukin-6 in bone metastasis and cancer progression. Eur J Cancer 46: 1223-1231, 2010. 